\title{
ON THE ENHANCEMENT OF MIXING AND DIFFUSION IN AN AXISYMMETRIC JET BY MEANS OF VORTEX GENERATORS
}

\author{
${ }^{* 1}$ Kensuke Miura, ${ }^{* 1}$ Koji Nagata, ${ }^{* 1}$ Yasuhiko Sakai, ${ }^{* 1}$ Osamu Terashima, and ${ }^{* 1}$ Yasumasa Ito \\ ${ }^{* 1}$ Graduate School of Engineering, Nagoya University \\ Furo-cho, Chikusa-ku, Nagoya, 464-8603, Japan \\ miura.kensuke@a.mbox.nagoya-u.ac.jp
}

\begin{abstract}
The promotion of mixing and diffusion in an axisymmetric jet is tried by using multiple prominences: six vortex generators (VGs).

The experimental instrument consists of the wind tunnel and the test section. The square jet is issued at the exit of wind tunnel. The circular skimmer of $30 \mathrm{~mm}$ diameter is placed at the inlet of the test section in order to eliminate boundary layer developed on the inner wall of the wind tunnel contraction. A VG has a right-angled triangle-shaped chip, and it is installed in an angle of 30 degrees from the flow direction. VGs are mounted around the exit of the skimmer and their motions can be controlled independently by computer programming. VGs can impose the disturbances on the turbulent jet. In this study, two moving-modes are tested beside the stationary mode: in the first mode, all the VGs are oscillated radially in phase (axisymmetric mode); and in the second mode, three VGs in the opposite side are put in and out to the main flow region alternately (alternative mode). Next, the experiment in a heated axisymmetric jet is performed where heat is considered as a passive scalar. Simultaneous measurements of the velocity and temperature are performed using a composite probe of I-type Hot-wire and I-type Cold-wire.

The results show the axisymmetric mode is the most effective for the promotion of the heat transport in the near-field region of the axisymmetric jet exit.
\end{abstract}

Keywords: Jet, Flow Control, Turbulent Flow, Vortex, Active Control

\section{INTRODUCTION}

Axisymmetric turbulent jet is one of the basic flows in the fluid mechanics, and it is widely applied to various industrial situations. Therefore, several techniques to control the jet flow were developed and tested in the previous studies.

Controlling of the jet flow with using tabs put around the jet exit to reduce the jet noise was performed by Bradbury et al.[1]. They showed that tabs could prevent the arising of the vortex ring at the jet effectively in the condition that two tabs were put in axisymmetric position to the center of the jet and the jet noise could be reduced. Rogers et al.[2] investigated the availability of longitudinal vortices to the enhancement of the mass entrainment and the reduction of the jet noise in a subsonic rectangular jet. They generated longitudinal vortex with using half-delta wings. And then they investigated the influence of the angles of attack and its shapes on the control by the flow visualization and noise measurements. Zaman et al.[3] investigated the influences of the shape of the jet exit on the spreading characteristic of subsonic and supersonic axisymmetric jet. They revealed that the lobed-shape nozzle suppressed the screech stage and spreading of the jet compared with other shapes in the supersonic condition. They also revealed that the existence of tabs around the jet exit enhanced its spreading in both supersonic and subsonic conditions. The heat diffusion in a heated axisymmetric jet with tabs just downstream of the jet exit was investigated by Mi et al.[4]. They measured the temperature field in the jet by means of a cold-wire thermometry and indicated that the existence of tabs distorted the jet flow and brought to cause the mean temperature to decrease more rapidly, implying an increase mixing rate.

In contrast, as an active control of the jet flow, Benard et al.[5] performed the control of an axisymmetric jet with using dielectric barrier discharge actuators that were installed along the lips of the diffuser. They revealed that the actuators could enhance the spreading of the jet, reduce the length of the jet core, and increase the turbulent kinetic energy along the jet centerline.

In this paper, to develop the innovative and effective control technique of the turbulent mixing in the jet flow, the experiments are performed in a heated axisymmetric jet which is controlled by vortex generators whose shape is half-delta wing. The velocity and temperature field in the jet under the three different controlling modes are measured simultaneously and the effect of the control on the turbulent mixing is discussed.

\section{EXPERIMENTAL SETUP}

\subsection{Axisymmetric jet}

Figure 1 shows a schematic view of the experimental apparatus and coordinate system of the present axisymmetric jet. A circular skimmer whose diameter $d$ is $30 \mathrm{~mm}$ is installed $8.0 \mathrm{~mm}$ downstream of the nozzle exit in order to elim- 


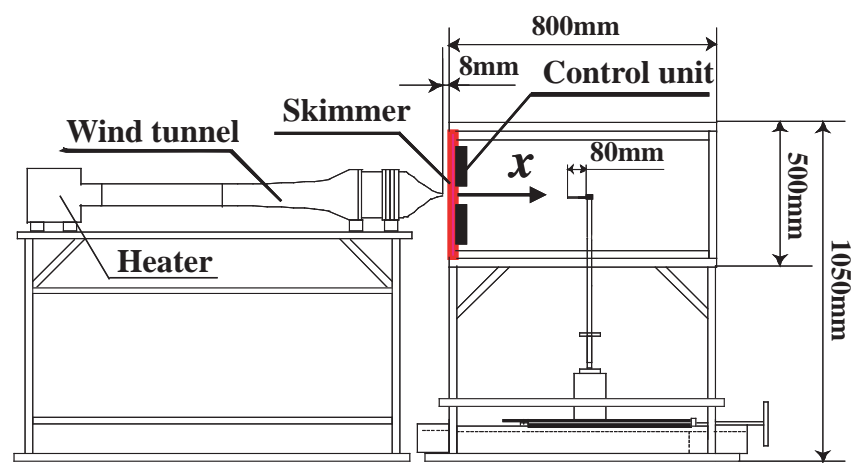

Fig. 1 Schematic of experimental apparatus

inate the boundary layer developed along the wall of the contraction nozzle of the wind tunnel. The coordinate system is as follows: the axial (streamwise) coordinate is $x$, the vertical (cross-streamwise) coordinate is $y$, and spanwise coordinate is $z$, and the origin of coordinate system is set at the center of the skimmer exit. A heated axisymmetric jet is realized using an electric heater set at the air intake of the blower of the wind tunnel. The temperature of the flow at the nozzle exit is controlled by means of a PID heater controller which has a thermocouple module fixed at the nozzle exit to moniter the temperature of the flow at the exit.

\subsection{Vortex generator}

In this study, half-delta wing prominences named vortex generator shown in Figure 2 is used to generate the longitudinal vortices into the initial shear layer of the jet. Six VGs are placed around the skimmer exit at regular intervals, so that their sharpest corner is heading toward to the upsteream direction with their attack angle becomes 30 degrees to the streamwise direction. VGs can move radial direction by means of six slide actuators connected to each VG and they can be controlled their motion individually. Maximum projection height $h$ of each VG are $3.75 \mathrm{~mm}$, and its solidity is $0.9 \%$.

\subsection{Measuring system and data acquisition}

Instantaneous velocity and temperature in the jet are simultaneously measured by means of a composite probe which consists of a hot-wire sensor and a cold-wire sensor shown in Figure 3. The cold-wire sensor is placed $2.0 \mathrm{~mm}$ upstream of the hot-wire sensor to eliminate the decline of the measurement accuracy of the cold-wire sensor caused by the heat disturbances of the hot-wire sensor. The hot-wire sensor and cold-wire sensor are operated by constant temperature anemometry and constant current thermometry, respectively. A tungsten fine wire whose diameter and length are $5.0 \mu \mathrm{m}$ and $1.0 \mathrm{~mm}$ respectively is used as the hot-wire sensor. In the same way, a tungsten fine wire whose diameter and length are $3.0 \mu \mathrm{m}$ and $2.0 \mathrm{~mm}$ respectively is used as the cold-wire sensor. The sampling frequency and sampling time of two sensors are $20 \mathrm{kHz}$ and about 26 seconds.

It should be noted here that there are two problems in the present measurement. One is the decline of the measurement accuracy of the velocity by means of the hot-wire sen-
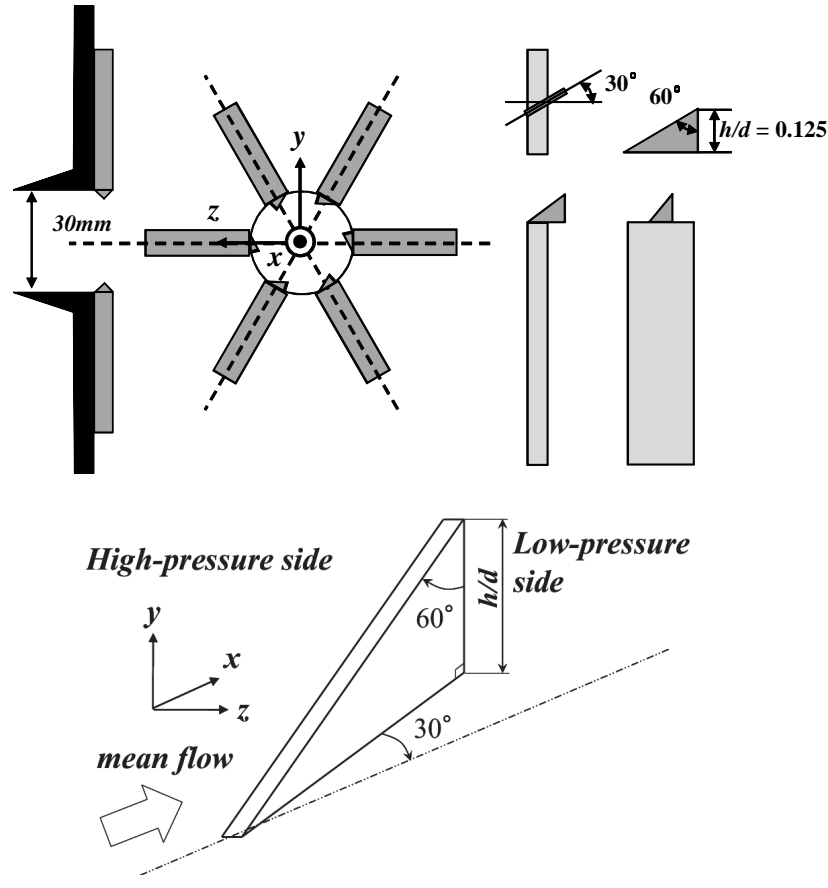

Fig. 2 Schematic of the vortex generator apparatus

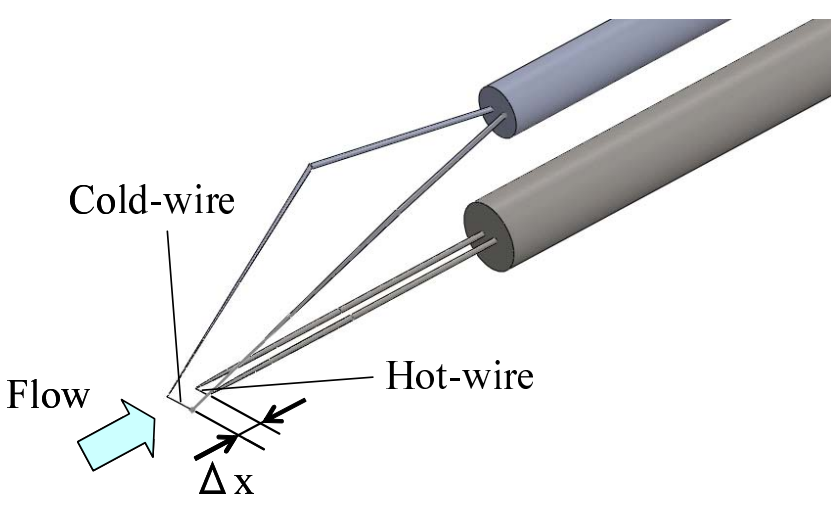

Fig. 3 Schematic of the composite probe

sor resulting from the varying of the temperature of the flow. The other is the arising of the temporal difference between the measurement result of the hot-wire sensor and cold-wire sensor resulted from the existence of the spatial gap for the streamwise direction between two sensors. Therefore, to keep the measurement accuracy, a temperature and temporal compensation of the measurement results of hot-wire sensor are digitally performed.

\subsection{Experimental condition}

The Reynolds number $\mathrm{R} e_{J}$ based on the diameter of the skimmer exit $d$, the streamwise velocity at the skimmer $U_{J}$ and the kinetic viscosity $v$ approximates 20,000 in the whole experiments. The temperature of the fluid at the skimmer exit $\theta_{J}$ is set about $7.5 \mathrm{~K}$ higher than that of ambient fluid $\theta_{a}$. It should be noted that the buoyancy of the jet is negligible and temperature of the fluid is considered as a passive scalar.

In this study, two moving-modes of VGs are tested beside 


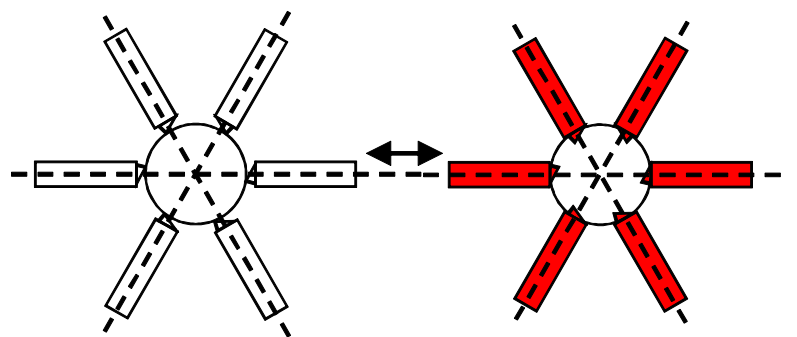

Axisymmetric mode

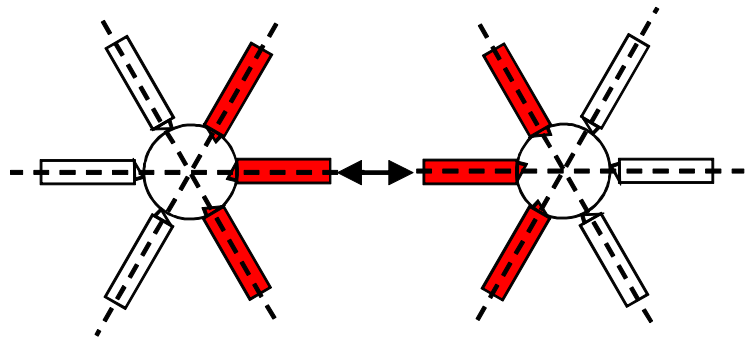

Alternative mode

Fig. 4 Moving pattern of the vortex generators.

The colored VGs are put out to the main flow.

the stationary mode: in the first mode, all VGs are oscillated radially in phase (axisymmetric mode: See upper side in Figure 4); and the second mode, three VGs in the opposite side are put in and out to the main flow region alternately (alternative mode: See lower side in Figure 4). The moving frequency of VGs in the axisymmetric mode and alternative mode is 3.0 $\mathrm{Hz}$.

\section{RESULTS AND DISCUSSIONS}

\subsection{Passive control (stationary mode)}

The contour maps of the turbulent heat flux normalized by $U_{J}$ and the thermal difference $\theta_{J}-\theta_{a},\langle u \theta\rangle / U_{J}\left(\theta_{J}-\theta_{a}\right)$ at $x / d=2.0$ without VGs and the stationary mode are shown in Figures 5 and 6 . Here \langle\rangle means time averaged value, $u$ is the instantaneous streamwise velocity fluctuation, and $\theta$ is the instantaneous temperature fluctuation. The ordinate and abscissa in Figure 5 and 6 are $z / d$ and $y / d$, respectively, and the color of the contour map shows the non-dimentional turbulent heat flux. It is found that the distribution of turbulent heat flux is distorted by the passive control. Further, it is also found that the turbulent heat flux near the jet centerline becomes higher than that without control.

\subsection{Active control (axisymmetric and alterna- tive control)}

Contour maps of the non-dimentional turbulent heat flux $\langle u \theta\rangle / U_{J}\left(\theta_{J}-\theta_{a}\right)$ at $x / d=2.0$ with axisymmetric mode and alternative mode are shown in Figures 7 and 8. The meanings of the ordinate, abscissa, and color of the contour map in Figure 7 and 8 are the same with those in Figure 5. It is found that the distribution of the turbulent heat flux is distorted in both controlling modes and the peak value of the turbulent heat flux in the measurement plane becomes higher than that in the stationary mode. Figure 7 shows that the higher turbulent heat flux is observed at both the inner and outer side of the jet, while Figure 8 shows it exist only in the inner side. Therefore, it can be concluded that the heat transport in the heated jet specifically depends on the active controlling mode of VGs.

\section{CONCLUSION}

In this paper, instantaneous velocity and temperature field are investigated in the heated axisymmetric turbulent jet in three controlling conditions with using half-delta wing prominences named vortex generators. The knowledge acquired in this research is written down below.

1. Turbulent heat flux in the jet can be controlled by VGs with passive control (stationary mode).

2. Turbulent heat flux in the jet can be also controlled by VGs with active control (axisymmetric and alternative mode). It is found that the heat transport on the jet is more enhanced by the active control compared with the passive control. Specifically the pattern of heat flux depends on the mode of the active control, and the axisymmetric mode is the most effective for the promotion of the heat transport in the near-field of the jet exit.

\section{ACKNOWLEDGEMENT}

The authors express their gratitude for partial financial support of this study by Grants-in-Aid for scientific research (No. 23760155).

\section{REFERENCES}

[1] Bradbury, L.J.S. and Khadem, A.H., "The Distortion of a Jet By Tabs", J. Fluid Mech. Vol. 70(1975), pp. 801-813

[2] Rogers C.B. and Parekh D.E., "Mixing Enhancement by and Noise Characteristics of Streamwise Vortices in an Air Jet”, AIAA J. Vol. 32(1994), pp. 464-471

[3] Zaman K.B.M.Q, "Spreading characteristics of compressive jets from nozzles of various geometries", J. Fluid Mech. Vol. 383(1999), pp. 197-228

[4] Mi J. and Nathan G.J, "Effect of small vortex-generators on scalar mixing in the developing region of a turbulent jet", Int. J. Heat and Mass Trans. Vol. 42(1999), pp. 3919-3926

[5] Benard N., Bonnet J. P., Touchard G., and Moreau E., "Flow Control by Dielectric Barrier Discharge Actuators: Jet Mixing Enhancement", AIAA J. Vol. 46(2008), pp. 2293-2305 
4th International Conference on Jets, Wakes and Separated Flows, ICJWSF2013

September 17-21, 2013, Nagoya, JAPAN

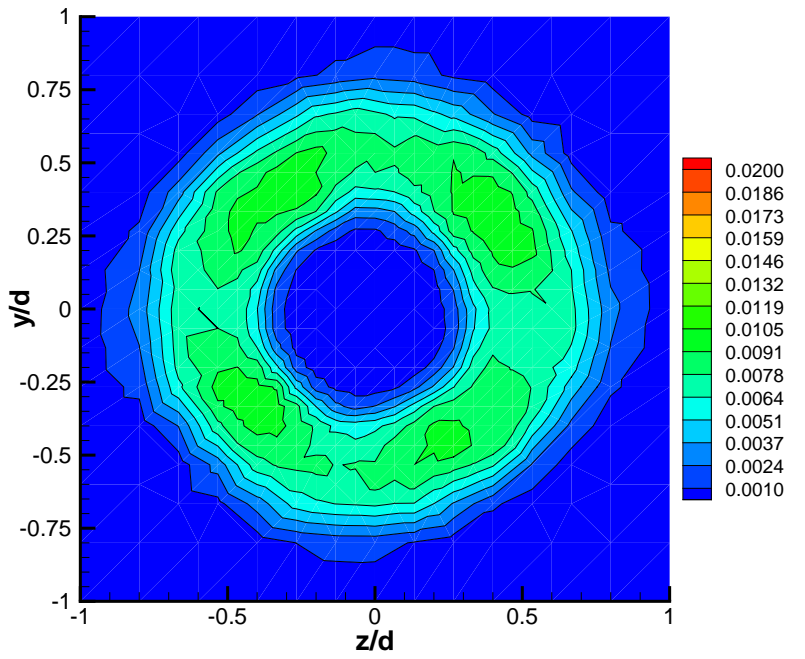

Fig. 5 Contour map of turbulent heat flux $\langle u \theta\rangle / U_{J}\left(\theta_{J}-\theta_{a}\right)$ distribution without VGs

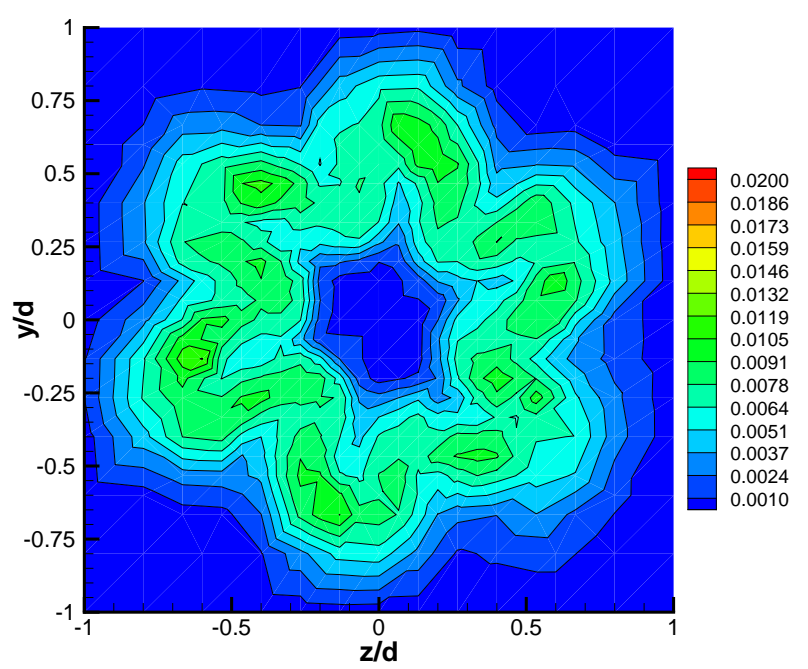

Fig. 6 Contour map of turbulent heat flux $\langle u \theta\rangle / U_{J}\left(\theta_{J}-\theta_{a}\right)$ distribution with static mode

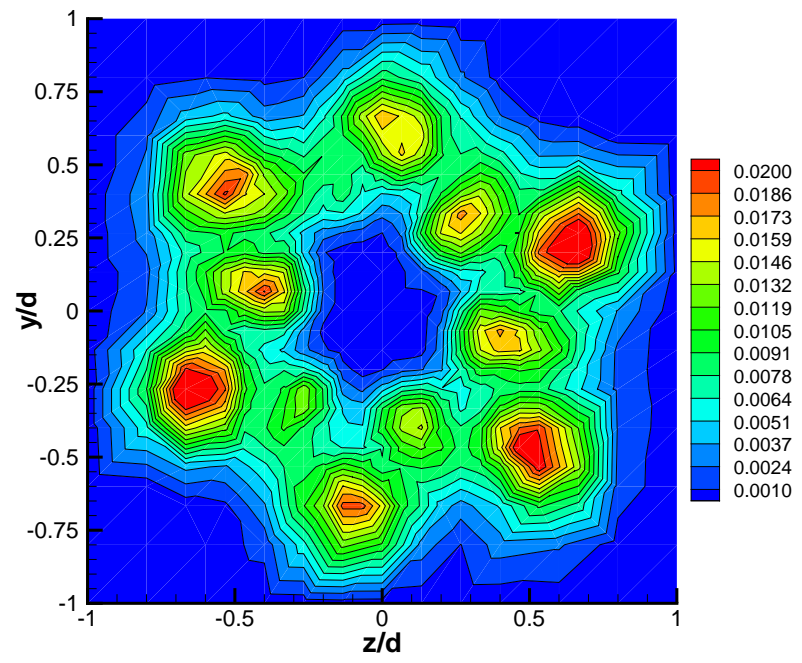

Fig. 7 Contour map of turbulent heat flux $\langle u \theta\rangle / U_{J}\left(\theta_{J}-\theta_{a}\right)$ distribution with axisymmetric mode

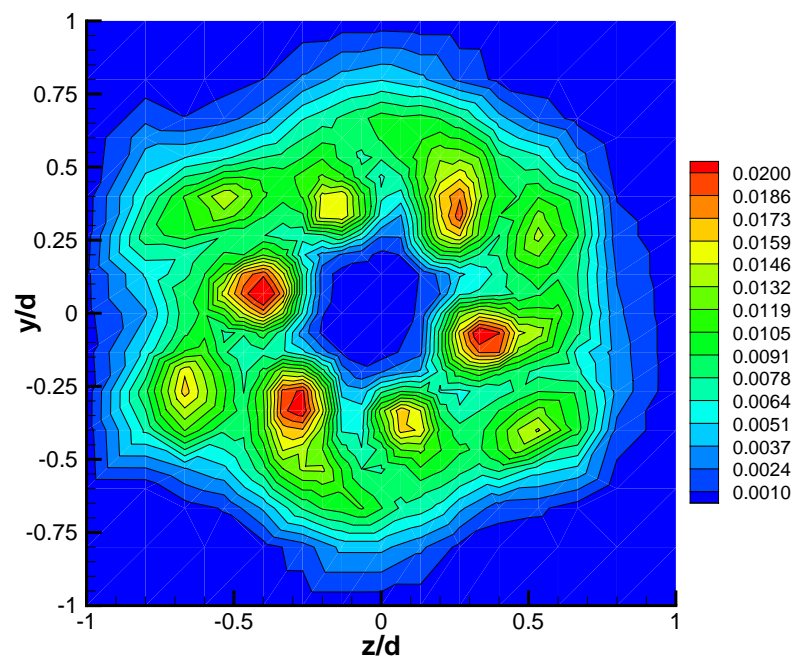

Fig. 8 Contour map of turbulent heat flux $\langle u \theta\rangle / U_{J}\left(\theta_{J}-\theta_{a}\right)$ distribution with alternative mode 